\title{
Mitteilungen der Deutschen Gesellschaft für Pathologie
}

Pathologe $2020 \cdot 41: 425-427$

https://doi.org/10.1007/s00292-020-00798-5

(c) Springer Medizin Verlag GmbH, ein Teil von Springer Nature 2020

Geschäftsstelle der Deutschen Gesellschaft für Pathologie e. V.

Jörg Maas (V.i.S. d.P.)

Generalsekretär der Deutschen Gesellschaft für Pathologie e. V. (DGP)

Robert-Koch-Platz 9, 10115 Berlin

Tel. 030-25 760-728 · Fax 030-25 760-729

E-Mail: geschaeftsstelle@pathologie-dgp.de

www.pathologie-dgp.de

\section{Pressemitteilung: An Corona Verstorbene sollten obduziert werden}

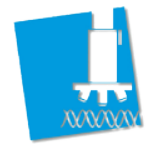

BUNDESVERBAND

DEUTSCHER

PATHOLOGEN E.V.

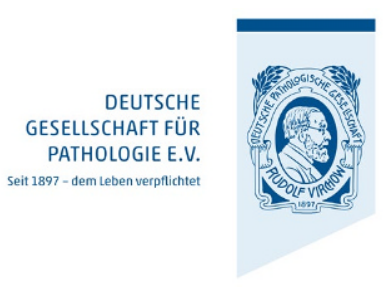

Berlin, 7. April 2020. Der Bundesverband Deutscher Pathologen (BDP) und die Deutsche Gesellschaft für Pathologie (DGP) fordern möglichst zahlreiche Obduktionen von Corona-Verstorbenen. Sie widersprechen damit der Empfehlung des Robert-Koch-Instituts (RKI), in diesen Fällen innere Leichenschauen $\mathrm{zu}$ vermeiden. Im Gegenteil sei es notwendig, weitere Erkenntnisse über die Erkrankung und deren oft erstaunlich fulminanten Verlauf zu gewinnen und offene Fragen zu beantworten.

Im besten Fall ließen sich daraus weitere Therapieoptionen ableiten - darin bestehe der Wert der Obduktion für die Lebenden, so Prof. Dr. med. K.-F. Bürrig, Präsident des Bundesverbandes. Die Obduktion sei in hohem öffentlichem Interesse und sollte deshalb nicht vermieden, sondern im Gegenteil so häufig wie möglich durchgeführt werden. Schon bei Ausbruch des Marburg-Virus, bei HIV, bei SARS, MERS und BSE haben Befunde aus der Pathologie und Neuropathologie geholfen, die klinischen Krankheitsbilder zu verstehen und haben damit auch therapeutische Konzepte beeinflusst. Dies muss auch für COVID-19 gelten. Dieses Anliegen hat auch Prof. Dr. T. Welte vom Deutschen Zentrum für Lungenforschung/DZL und Direktor der Klinik für Pneumologie und Infektionsmedizin der Med. Hochschule Hannover/MHH an die DGP gerichtet.

In der RKI-Empfehlungen vom 24.03.2020 heißt es u.a.: „Eine innere Leichenschau, Autopsien oder andere aerosolproduzierende Maßnahmen sollten vermieden werden. Sind diese notwendig, sollten diese auf ein Minimum beschränkt bleiben." Diese Empfehlung richte, so Bürrig, das Augenmerk auf die Vermeidung von infektionsgefährlichen Aerosolen bei der Leichenöffnung. Das sei ein wichtiger Aspekt, aber als Entscheidungsgrundlage zu schmal. Zumal bei den Obduktionen nach allen einschlägigen Vorgaben der Schutz des medizinischen und nicht-medizinischen Personals sichergestellt wird. BDP und DGP bitten das RKI darum, die Gesundheitsbehörden entsprechend zu informieren.
An der Rheinisch-Westfälischen Technischen Hochschule Aachen werde zudem gerade ein Register für COVID-19-Obduktionen im deutschsprachigen Raum eingerichtet, so der Vorsitzende der DGP, Prof. Dr. Gustavo Baretton. In Aachen werden die Obduktionsinformationen gesammelt. Die dezentrale Asservierung von Untersuchungsgewebe stellt sicher, dass es für Spezialuntersuchungen zur Ver- fügung steht. DGP und BDP planen einen schnellen Wissenstransfer nicht nur innerhalb des Fachs Pathologie, sondern gerade auch an Lungenfachärzte sowie Intensivmediziner, und ebenso an die zuständigen Behörden.

Die Pressemitteilung hat eine hohe Resonanz in den Medien erfahren.

Weitere Informationen unter: www.pathologie-dgp.de/die-dgp/ aktuelles 
Sommer, Ulrich'; Baretton, Gustavo ; ; Hartmann, Arndt'² Eckstein, Markus'; Schwamborn, Kristina3; Weichert, Wilko3; Kristiansen, Glen ${ }^{4}$; Wild, Peter ${ }^{5}$; Knuechel-Clarke, Ruth ${ }^{6}$; Schirmacher, Peter7; Dietel, Manfred ${ }^{8,9}$; Jöhrens, Korinna ${ }^{1,9}$

${ }^{1}$ Institut für Pathologie, Universitätsklinikum Carl Gustav Carus, TU Dresden,

${ }^{2}$ Institut für Pathologie, Universitätsklinikum Erlangen, FriedrichAlexander-Universität Erlangen-Nürnberg

${ }^{3}$ Institut für Pathologie, TUM, Universitätsklinikum München,

${ }^{4}$ Institut für Pathologie, Universitätsklinikum Bonn,

${ }^{5}$ Institut für Pathologie, Universitätsklinikum Frankfurt, Johann

Wolfgang von Goethe Universität

${ }^{6}$ Institut für Pathologie, Universitätsklinikum Aachen,

${ }^{7}$ Institut für Pathologie, Universitätsklinikum Heidelberg,

${ }^{8}$ Institut für Pathologie, Universitätsklinikum Charité Berlin

${ }^{9}$ QuiP GmbH, Berlin

\section{Ergebnisse des ersten prototypischen Ringversuchs der QuIP zur PD-L1 Expression in Urothelkarzinomen}

Im Gegensatz zu nicht-kleinzelligen Lungenkarzinomen (NSCLC) ist bei Urothelkarzinomen zur Stratifizierung für eine Therapie mit Checkpointinhibitoren nicht nur die PD-L1 Expression der Tumorzellen, sondern auch der Immunzellen entscheidend. Die Zulassungen für Atezolizumab und Pembrolizumab fordern unterschiedliche Scores und Cut-Offs. Für die Therapieentscheidung bei Atezolizumab ist die Expression der Immunzellen (IC) mit einem Cut-Off von $\geq 5 \%$ essentiell, für die Therapieentscheidung bei Pembrolizumab ist die Expression von PD-L1b auf den Immunzellen und Tumorzellen in Form eines kombiniert erhobenen Scores (CPS) mit einem Cut-Off von $\geq 10$ maßgeblich. Da in der Regel bei Diagnosestellung das intendierte Therapeutikum nicht bekannt ist, müssen beide Scores angegeben werden. Daher wurde von der QualitätssicherungsInitiative Pathologie (QuIP) ein prototypischer Ringversuch (RV) erarbeitet, der beide therapierelevanten Scores berücksichtigte.

\section{Aufbau des RV}

Für den IC und den CPS wurden entsprechend der Zulassungen getrennte Kategorien definiert

IC

- Kategorie 1: $<5 \%$

- Kategorie 2: $\geq 5 \%$

CPS

- Kategorie 1: $<1$

- Kategorie 2: $\geq 1<10$

- Kategorie $3 \geq 10$

Die Kategorien 1 und 2 des CPS wurden bei der Auswertung zusammengefasst, da die Kategorie 1 entsprechend der Zulassung der EMA zum Zeitpunkt des offenen Ringversuches für die Therapieentscheidung keine Rolle mehr spielte.

\section{Vorbereitung des Ringver- suchs}

Als Lead-Panelinstitute fungierten die Institute für Pathologie der Universitäten Dresden (organisatorisch führend), München und Erlangen. Die Pathologien der Universitätskliniken Aachen,
Tab. 1 Häufigkeit der Antikörper unter allen Teilnehmern

\begin{tabular}{lll} 
Klon & Hersteller & $\begin{array}{l}\text { Anzahl der Teilneh- } \\
\text { mer }\end{array}$ \\
\hline CAL10 & Zytomed & 20 \\
22C3 & Agilent & 16 \\
$28-8$ & Abcam & 9 \\
E1L3N & Cell Signaling & 11 \\
SP263 & Roche & 8 \\
ZR3 & Zeta corp & 4 \\
QR1 & Quartett & 8 \\
SP142 & Roche & 4 \\
Andere & & 2
\end{tabular}

Tab. 2 Wahl der Plattform

\begin{tabular}{ll} 
Plattform (Hersteller) & Anzahl der Teilnehmer \\
\hline Ventana (Roche) & 27 \\
Dako (Agilent) & 21 \\
Leica & 13 \\
Intellipath (Zytomed Systems) & 10 \\
Thermo Scientific & 3 \\
Andere & 7 \\
Keine Angabe & 1
\end{tabular}

Bonn, Frankfurt und Heidelberg unterstützten den Ringversuch als Panelinstitute.

Es wurden insgesamt 27 Fälle (von Zystektomiepräparaten und transurethralen Resektaten von Urothelkarzinomem) für den internen RV von den 3 Lead-Panelinstituten zur Verfügung gestellt. Nach Testung der Fälle durch die Lead- und Panelinstitute und Auswertung der Ergebnisse wurden 10 Fälle für den offenen RV identifiziert.

Für jeden Score wurde ein Grenzfall (diese Färbeergebnisse lagen in der Nähe eines Cut-offs) gewählt. Die übrigen 9 ausgewählten Fälle wurden entweder einheitlich oder von max. einem Institut abweichend interpretiert.

\section{Ergebnisse des Ringver- suchs}

An dem RV nahmen 82 Institute teil. Die Durchführung des RV erfolgte im Zeitraum vom 04.04.-03.05.2019.
Für jeden Fall wurden zwei Punkte pro Score vergeben. Die Bestehensgrenze lag bei 18 Punkten pro Score. Sofern die Schnitte nach der Durchführung der Immunhistologie nachweislich technisch nicht auswertbar waren und dies vom Teilnehmer so auch kundgetan wurde, wurde ein Punkt vergeben, bei inkorrekter Zuordnung zu den Kategorien erhielten die Teilnehmer keinen Punkt.

Für den CPS konnte 50 (61 \%) Instituten eine erfolgreiche Teilnahme bescheinigt werden. Wohingegen 32 (39\%) Institute weniger als 18 Punkte erzielten und damit am Ringversuch ohne Erfolg teilnahmen. Unter Berücksichtigung der Lead- und $\mathrm{Pa}$ nelinstitute bestanden den CPS Score 57 (64\%) Einrichtungen. An dem IC-Teil nahmen 51 (62\%) Einrichtungen mit Erfolg teil. $38 \%$ der Institute (31) konnte nur die Teilnahme bescheinigt werden. Unter Einbeziehung der Lead- und Panelinstitute, lag die 
Tab. 3 Antikörper und Bestehensquoten von CPS und IC

\begin{tabular}{lll} 
KIon & CPS $(\%)$ & IC $(\%)$ \\
\hline CAL10 & $16 / 20(80)$ & $15 / 20(75)$ \\
$22 C 3$ & $11 / 16(68,8)$ & $10 / 16(62,5)$ \\
E1L3N & $7 / 11(63,6)$ & $7 / 11(63,6)$ \\
$28-8$ & $7 / 9(77,8)$ & $7 / 9(77,8)$ \\
SP263 & $7 / 8(87,5)$ & $7 / 8(87,5)$ \\
QR1 & $4 / 8(50)$ & $8 / 8(100)$ \\
SP142 & $2 / 4(50)$ & $3 / 4(75)$ \\
ZR3 & $4 / 4(100)$ & $3 / 4(75)$
\end{tabular}

Bestehensquote des IC Scores bei $65 \%$ (58).

Beide Scores bestanden 37 (45\%) Teilnehmer; werden die Lead und Panelinstitute hinzugenommen, liegt die kombinierte Bestehensquote bei $49 \%$ (44 Einrichtungen).

\section{Re-Evaluation der zurück- gesandten Schnitte}

Nach der Re-Evaluation aller Schnitte von allen Teilnehmern durch drei Fachärzte des führenden Lead-Panelinstitutes zeigte sich, dass unabhängig von den verschiedenen Cut-Offs 21 Teilnehmer Probleme mit der Färbequalität, 22 Teilnehmer ein Problem bei der Interpretation der Fälle und 24 Teilnehmer sowohl Probleme bei der Färbung als auch bei der Interpretation hatten. Die Färbeprobleme beruhten ausschließlich auf einer schwächeren Anfärbung der Tumor -und/oder Immunzellen im Vergleich zu den Färbeergebnissen der Lead- und Panelinstitute.

\section{Eingesetzte Primäranti- körper}

Die Wahl des eingesetzten Primärantikörpers war den teilnehmenden Einrichtungen freigestellt. Die von den Teilnehmern eingesetzten Primärantikörper sind in $\bullet$ Tab. 1 aufgeführt.

Die eingesetzte Plattform war nicht vorgeschrieben. - Tab. 2 zeigt das Verteilungsmuster der verwendeten Plattformen nach Herstellern sortiert.
- Tab. 3 zeigt die Anzahl der Teilnehmer und die Bestehensquote bezogen auf den jeweils verwendeten Antikörper (berücksichtigt wurden nur die Teilnehmer mit Färbeproblemen).

\section{Diskussion}

Der prototypische Ringversuch „PD-L1 Expression in Urothelkarzinomen" zeigt, dass weiterhin viele Pathologen Interesse an Qualitätssicherung haben. In Deutschland ist offenbar die Etablierung von LDT bei PD-L1 weit verbreitet. Es ist zu erkennen, dass, wie bei dem PD-L1 Ringversuch Lunge, eine Etablierung außerhalb der Kits mit gutem diagnostischen Ergebnis möglich ist und die Anwendung der Kits somit keine notwendige Voraussetzung für die korrekte PD-L1 Analyse darstellt. Dennoch lassen sich aus diesem Ringversuch verschiedene Tendenzen bezogen auf den Einsatz der Primärantikörper ableiten. Zum einen zeigt sich, dass mit den Primärantikörpern SP142 und QR1 der korrekte CPS schwerer ermittelbar ist, als mit den übrigen Antikörpern. Weiterhin scheinen die Antikörper E1L3N, 28-8 und SP263 für die Ermittlung beider Scores von vergleichbar guter Performanz zu sein.

Dennoch ist bei diesem Ringversuch auch erkennbar, dass sowohl der CPS als auch der IC eine Herausforderung für die Teilnehmer darstellen und weitere Schulungen für die korrekte Interpretation unbedingt notwendig sind. 